CLINICAL STUDY

\title{
Radioactive iodine in the treatment of medullary thyroid carcinoma: a controlled multicenter study
}

\author{
J A A Meijer ${ }^{1}$, L E H Bakker², G D Valk ${ }^{3}$, W W de Herder ${ }^{4}$, J H W de Wilt ${ }^{5}$, R T Netea-Maier ${ }^{6}$, N Schaper ${ }^{7}$, E Fliers ${ }^{8}$, \\ P Lips ${ }^{9}$, J T Plukker ${ }^{10}$, T P Links ${ }^{11}$ and J A Smit ${ }^{6}$ \\ ${ }^{1}$ Department of Internal Medicine, Albert Schweitzer Hospital, Albert Schweitzerplaats 25, 3318 AT Dordrecht, The Netherlands, ${ }^{2}$ Departments of \\ Endocrinology and Metabolic Diseases, Leiden University Medical Center, 2300 RC Leiden, The Netherlands, ${ }^{3}$ Department of Internal Medicine and \\ Endocrinology, University Medical Center, Utrecht, 3508 GA Utrecht, The Netherlands, ${ }^{4}$ Section of Endocrinology, Department of Internal Medicine, \\ Erasmus Medical Center, 3015 CE Rotterdam, The Netherlands, Departments of ${ }^{5}$ Surgery and ${ }^{6}$ Endocrinology, Radboud University Nijmegen Medical \\ Center, 6500 HB Nijmegen, The Netherlands, ${ }^{7}$ Department of Endocrinology, Maastricht University Medical Center, 6200 MD Maastricht, The \\ Netherlands, ${ }^{8}$ Department of Endocrinology and Metabolism, Academic Medical Center, University of Amsterdam, 1100 DE Amsterdam, The Netherlands, \\ ${ }^{9}$ Department of Endocrinology, VU Medical Center, Amsterdam, $1007 \mathrm{MB}$ Amsterdam, The Netherlands, Departments of ${ }^{10}$ Surgery and ${ }^{11}$ Endocrinology, \\ University Medical Center Groningen, 9700 RB Groningen, The Netherlands
}

(Correspondence should be addressed to J A A Meijer; Email: jaa.meijer@asz.nl)

\begin{abstract}
Objective: Radioactive iodine (RAI) therapy in medullary thyroid carcinoma (MTC) is applied in some centers, based on the assumption that cross-irradiation from thyroid follicular cells may be beneficial. However, no systematic studies on the effect of RAI treatment in MTC have been performed. The aim of this study was to analyze the effect of RAI treatment on survival in MTC patients.

Design: Retrospective multicenter study in eight University Medical Centers in The Netherlands.

Methods: Two hundred and ninety three MTC patients without distant metastases who had undergone a total thyroidectomy were included between 1980 and 2007. Patients were stratified by clinical appearance, hereditary stage, screening status, and localization. All patients underwent regular surgical treatment with additional RAI treatment in 61 patients. Main outcome measures were disease-free survival (DFS) and disease-specific survival (DSS). Cure was defined as biochemical and radiological absence of disease.

Results: In multivariate analysis, stratification according to clinical appearance $(P=0.72)$, hereditary stage $(P=0.96)$, localization $(P=0.69)$, and screening status $(P=0.31)$ revealed no significant effects of RAI treatment on DFS. Multivariate analysis showed no significant difference in DSS for the two groups stratified according to clinical appearance $(P=0.14)$. Owing to limited number of events, multivariate analysis was not possible for DSS in the other groups of stratification.

Conclusions: Based on the results of the present analysis, we conclude that RAI has no place in the treatment of MTC.
\end{abstract}

European Journal of Endocrinology 168 779-786

\section{Introduction}

Curative treatment of medullary thyroid carcinoma (MTC) consists of a total thyroidectomy and central neck dissection with additional lymph node dissection of lateral compartments when lymph node metastases are proven or suspected $(1,2,3,4)$. In $\sim 75 \%$ of patients, MTC is sporadic and in $25 \%$ the disease is genetically inherited (either solitary or in the context of MEN2A or MEN2B).

In general, the prognosis is relatively favorable with a 10-year disease-specific survival (DSS) of $75 \%$ $(5,6,7,8)$. Besides calcitonin doubling time $(9,10$, $11,12)$, TNM stage is the most important prognostic factor with 10-year survival rates for stages I, II, III, and IV of 100, 93, 71 , and $21 \%$ respectively (13).
In contrast to the follicular cell in non-medullary differentiated thyroid carcinoma, parafollicular C-cells do not accumulate iodine. Therefore, radioactive iodine (RAI) therapy is not routinely used in the management of MTC.

The effect of RAI treatment in MTC patients has been investigated in several studies and showed contradictory results. Whereas in some studies no added value was found $(13,14,15)$, in one study with MTC patients and in several animal and in vitro studies, a beneficial effect of RAI in MTC $(16,17,18,19,20,21,22,23)$ was claimed. The effect, if present, is hypothesized to be due to the so-called bystander effect. Follicular cells, remaining in the thyroid bed after total thyroidectomy, may trap a sufficient amount of RAI destroying adjacent non-iodine-trapping cancer cells by the beta emission 
$(16,24)$. Elimination of remaining C-cells is particularly important in hereditary MTC as every C-cell is capable of malignant degeneration. Consequently, this could be an argument in favor of implementing RAI in the treatment of hereditary MTC. Nevertheless, the before mentioned studies are characterized by methodological limitations such as a small number of patients, limited follow-up duration, and lack of a control group.

The aim of this multicenter study was to analyze the effect of RAI treatment on the DFS and DSS in MTC patients without distant metastases, focusing on hereditary MTC. Given the multifocality and lifelong risk of malignant transformation of C-cells, advantage of RAI treatment would mainly be expected in patients with hereditary MTC. In addition, we hypothesized that if RAI has a beneficial effect, this would be restricted to patients with MTC limited to the thyroid (T1-3NOMO).

\section{Materials and methods}

In The Netherlands, MTC patients are generally treated in one of the University Medical Centers (centers). The aforementioned lack of consensus has in the past led to diversity in the application of RAI in the treatment of MTC in The Netherlands. Owing to this diversity, it was possible to assess the impact of RAI on the prognosis of patients with MTC in a retrospective multicenter study.

Patients were identified from hospital administrations and the national database of the Dutch pathology laboratories (PALGA). All patients diagnosed with MTC in one of the eight centers in The Netherlands since 1980 were identified and registered in the national database of the academic collaboration on MTC. The following are registered in this database: data on clinical characteristics; laboratory, histological, and radiological investigations; additional treatment modalities; and follow-up.

In the present analysis, patients diagnosed between 1980 and 2007 were included when MTC was histologically proven, a total thyroidectomy was performed, and clinical data were available for analysis. Patients were excluded if MTC was of medullarfollicular mixed type, follow-up was $<6$ months, distant metastases were present at presentation or within 6 months after primary surgery, or when primary surgery was not performed with curative intent. Historically, in some centers, RAI was administered according to the insight of the treating physician, some physicians referring all MTC patients for RAI, other physicians only referring hereditary MTC patients.

In order to study the hypothesis that RAI would be most beneficial in hereditary MTC, patients were stratified in hereditary MTC (proven by genetic analysis and/or a family history for MTC, MEN2A, or MEN2B) or sporadic MTC. As it is likely that the prognosis of patients with preclinical MTC or clinically apparent MTC is not comparable, patients were stratified according to this classification. Within the group of patients with clinically apparent MTC, a subanalysis of patients with hereditary MTC was performed.

Clinically apparent MTC was defined according to the American Thyroid Association (ATA) guideline, as a thyroidal node with a fine-needle aspiration or serum calcitonin diagnostic or suspicious for MTC (predominantly sporadic MTC) or a nodule $>5 \mathrm{~mm}$ on ultrasound at the moment of screening in hereditary MTC. A member from a family with hereditary MTC with a positive genetic or calcitonin screening and a thyroidal node $<5 \mathrm{~mm}$ on ultrasound was classified as preclinical MTC. Screening was defined as a member from a family with hereditary MTC referred for investigation of the presence of MTC. As we hypothesized that the potential effect of RAI treatment on disease (free) survival is to be expected in patients with MTC localized within the thyroid, patients were also stratified in T1-3NO (intrathyroidal) MTC or extrathyroidal (T4 and/or N1) (patients with distant metastases at diagnosis or within 6 months after operation were excluded). Besides, to correct for a possible advantage in prognosis, patients with hereditary clinically apparent MTC were stratified according to whether they had been diagnosed by screening or not. Patients were categorized in regular treatment (control group) and additional RAI treatment (RAI group).

TNM staging was classified according to the classification of the International Union Against Cancer (UICC) 6th edition based on pTNM. For the classification of intrathyroidal and extrathyroidal MTC, the 5th edition of the UICC was used because of the more distinct definition of $\mathrm{T}$ stage. $\mathrm{T}$ stage was determined on the basis of the pathology report; in case of multifocality, the largest MTC localization was used. N stage was based on the pathology report. NO was defined as the absence of metastases in the dissected lymph nodes from the central compartment (level 6) and, when applicable, the lateral compartment (level 2-5). After primary surgical intervention, basal (non-stimulated) calcitonin was measured frequently during the followup. In the presence of biochemical evidence of disease, imaging studies (including: neck ultrasound; CT scan of the neck, thorax, and abdomen; bone scan; or functional imaging ( $\mathrm{Tc}^{99}{ }^{-}$, MIBG-, somatostatin-, ${ }^{18}$ FDG-PET-, or ${ }^{18}$ F-DOPA-PET-scan) were performed.

Primary outcome variables were disease-free survival (DFS) and disease-specific mortality. The number of patients who underwent a re-intervention was a secondary outcome variable.

Cure was defined as the absence of biochemical and radiological evidence of disease including repeated basal calcitonin levels below the functional detection limit of the used assay (25). Recurrence was defined as biochemical and/or radiological evidence of disease after cure had been established. Biochemical recurrence was defined as calcitonin levels that, after a period of biochemical cure, were repeatedly above the functional detection limit of the assay, after exclusion of other causes of calcitonin elevation. 
Death causes were investigated using medical records, death certificates, and autopsy records. The disease-specific mortality was defined when the patient had progressive metastatic or relapsing MTC without other potentially fatal disease and/or when MTC was mentioned as death cause in the death certificate or autopsy file. In all other cases, the patient died due to a non-MTC-specific cause.

Adequacy of the surgical intervention was retrospectively determined according to the most recent ATA guidelines of 2009 (4). In this analysis, a central neck dissection (level VI) was defined adequate, whereas in the pathology report, at least two lymph nodes were described in this compartment.

\section{Statistical analysis}

Statistical analysis was performed using the Predictive Analytics Software (PASW) Statistics, version 18.0 (Chicago, IL, USA). Categorical data were presented as absolute values and proportions. Quantitative data were presented as the mean \pm s.D. in case of a symmetrical distribution or the median (range) for asymmetrical distribution. Differences in patient characteristics were analyzed by (unpaired) $t$-test, Mann-Whitney $U$ test for non-normally distributed data, or $\chi^{2}$-test (for proportions). Correction for confounding on DSS and DFS was performed using a Cox regression analysis according to the association model (26). In this model, the independent variable was DFS or DSS. The change in regression coefficient $(\Delta \beta)$ of the variable 'ablation with RAI' was evaluated in a model without and with the possible confounder. The strongest confounder was then used in a multiple regression model. Confounding was considered relevant if the change in regression coefficient was at least $10 \%$. All confounders, together with the primary variable 'ablation with RAI', were then used in a multivariable Cox regression analysis to analyze the DFS and DSS. A $P$ value of $<0.05$ was considered statistically significant. Outcomes are presented with hazard ratios (HRs) with accompanying $P$ values and 95\% CI. The following variables were analyzed for possible confounding: age, gender, hereditary stage, center, tumor extension (TNM), localization, adequacy of surgery, and in the group of patients with hereditary clinically apparent MTC also screening status. In addition, pT stage was included as covariable for tumor extension.

\section{Results}

\section{Patient characteristics}

In the national database of the academic collaboration on MTC, a total of 490 patients with MTC have been registered since 1980. For the current analysis, 197 patients were excluded (see Fig. 1). Of the included 293 patients, 232 underwent regular surgical treatment (control group) and 61 patients underwent regular surgical treatment with additional RAI treatment (RAI group). The patients in the RAI group were treated in five different centers contributing 2, 4, 10,22 , and 23 patients respectively. Patient characteristics are shown in Table 1.

Clinically apparent MTC MTC was clinically apparent in 230 patients of whom 179 patients underwent regular surgical treatment (control group) and the remaining 51 underwent regular surgical treatment with additional RAI treatment (RAI) group. The median follow-up duration of these patients was 116.6 (range 7-372) months. During the follow-up, $21.7 \%$ of

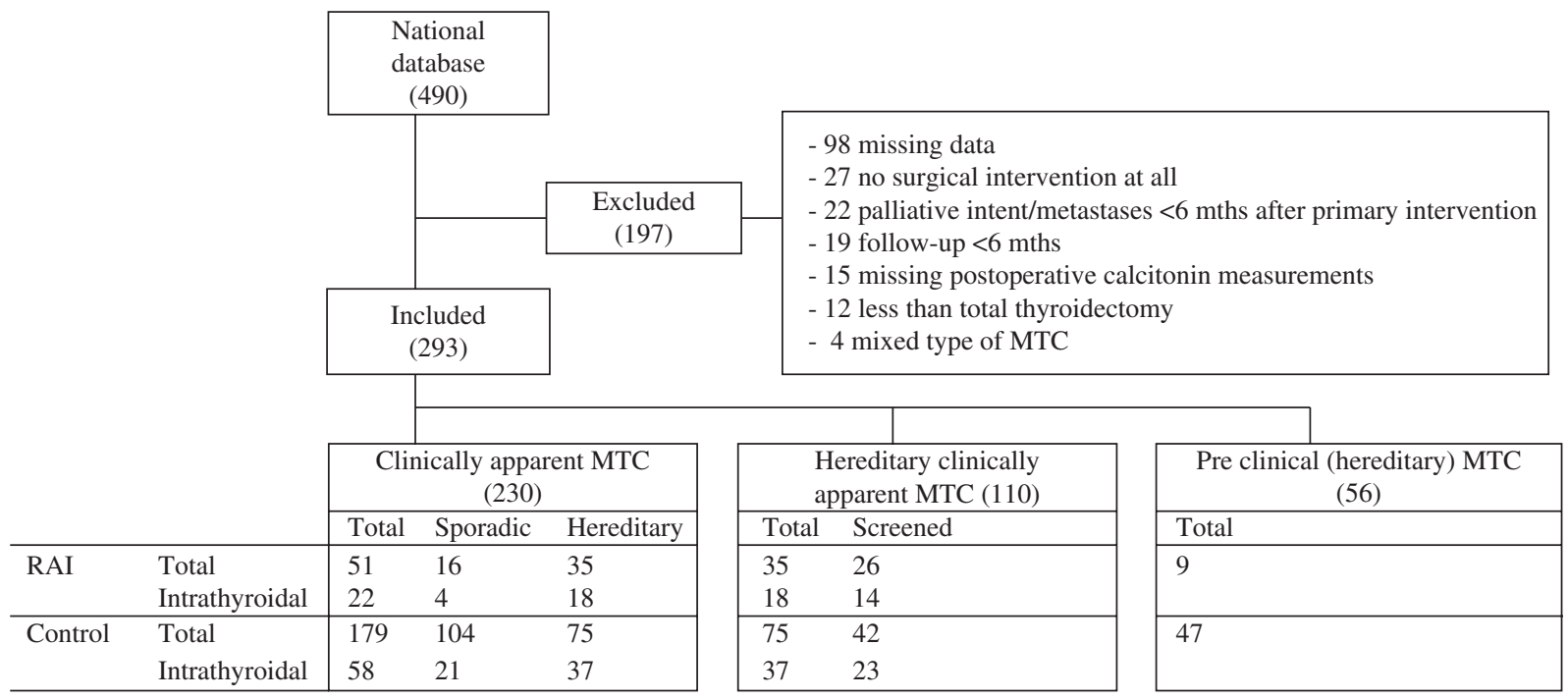

Figure 1 Study enrollment flow diagram. 


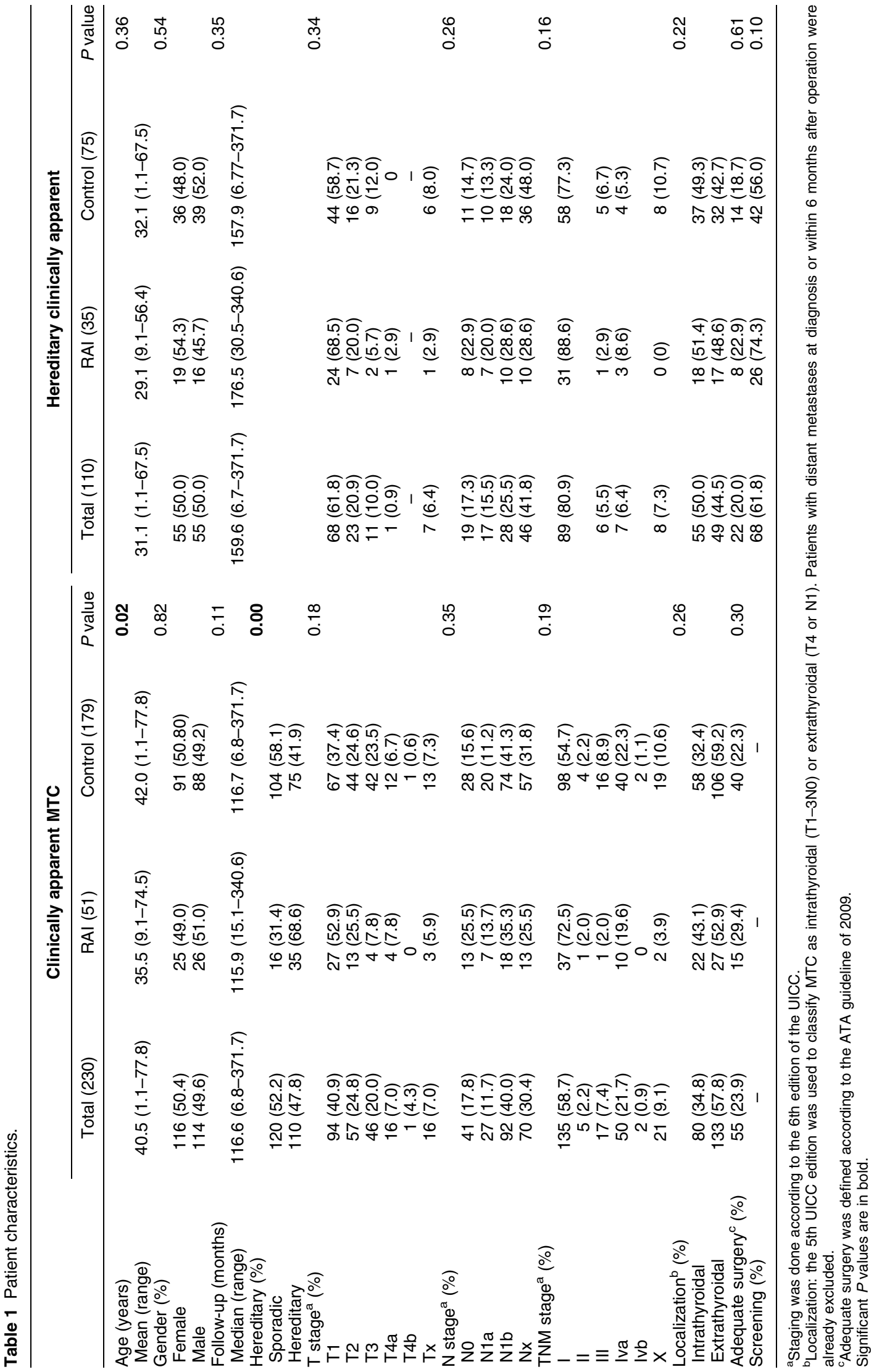


patients had a remission and recurrence occurred in $7.4 \%$. In $64.3 \%$ of the patients, calcitonin was detectable continuously during the follow-up and consequently could not be formally classified as cured. $6.5 \%$ of the patients could not be classified into recurrent or persistent MTC due to missing calcitonin values in the first period after the primary surgical intervention. Twenty-two patients $(9.6 \%)$ died from MTC, 14 patients died (6.1\%) due to other causes, and in eight patients $(3.5 \%)$ the cause of death was unclear.

There were no significant differences in patient characteristics between the control group and the RAI group (see Table 1) except for hereditary stage and age. In the RAI group, the hereditary type was present in $68.6 \%$ compared with $41.9 \%$ in the control group $(P=0.001)$. Mean age of patients in the RAI group was 35.5 years compared with 42.0 years in the control group $(P=0.02)$.

Hereditary clinically apparent MTC One hundred and ten patients had a hereditary clinically apparent MTC, of which 35 were treated with RAI and the remaining patients underwent regular treatment. The median follow-up duration of these patients was 159.6 (range 7-372) months. During the follow-up, 24.5\% of patients had a remission and recurrence occurred in $11.8 \%$. In $56.4 \%$ of the patients, calcitonin was detectable continuously during the follow-up. Owing to missing calcitonin values in the first period after the primary surgical intervention, $7.3 \%$ of the patients could not be classified into recurrent or persistent MTC. Five patients $(4.5 \%)$ died due to MTC and five patients died $(4.5 \%)$ due to other causes. There were no significant differences in patient characteristics between the control group and the RAI group (see Table 1).

Preclinical MTC Fifty-six patients were screened because of hereditary MTC and were diagnosed with preclinical MTC. Nine of these patients underwent additional treatment with RAI.

Mean age was 17.6 (range 2.3-53.9) years. Twentyeight patients $(50 \%)$ were female and the median follow-up duration of these patients was 154.0 (range 30-369) months. In 25 patients (44.6\%), the surgical intervention was done according to the ATA guideline. During the follow-up, $66.1 \%$ of patients had a remission and recurrence occurred in $0.5 \%$. In $16.1 \%$ of the patients, calcitonin was detectable continuously during the follow-up. A total of $10.6 \%$ of the patients could not be classified into recurrent or persistent MTC due to missing calcitonin values after primary surgical intervention. There were no significant differences in age, gender, follow-up duration, and number of patients treated according to the ATA guideline between the control group and the RAI group (data not shown).

\section{Disease-free survival}

Clinically apparent MTC During the follow-up, remission was obtained in $20.7 \%$ of patients in the control group and $25.5 \%$ of patients in the RAI group $(P=0.23)$. Recurrence occurred in 6.1 and $11.8 \%$ in the control and RAI groups respectively $(P=0.23)$. Univariate analysis showed no significant difference in DFS for the two groups (HR=0.91 HR for RAI; 95\% CI $0.63-1.32 ; P=0.63$; see Table 2).

Table 2 Univariate and multivariate Cox regression analysis of disease-free and disease-specific survival in patients with MTC comparing patients who underwent regular treatment (control group) and those who underwent regular treatment with additional RAI treatment (RAl group). Patients were stratified according to the following groups: clinically apparent MTC, clinically apparent hereditary MTC (with subanalysis of patients with intrathyroidal MTC and patients diagnosed by screening), and preclinical MTC.

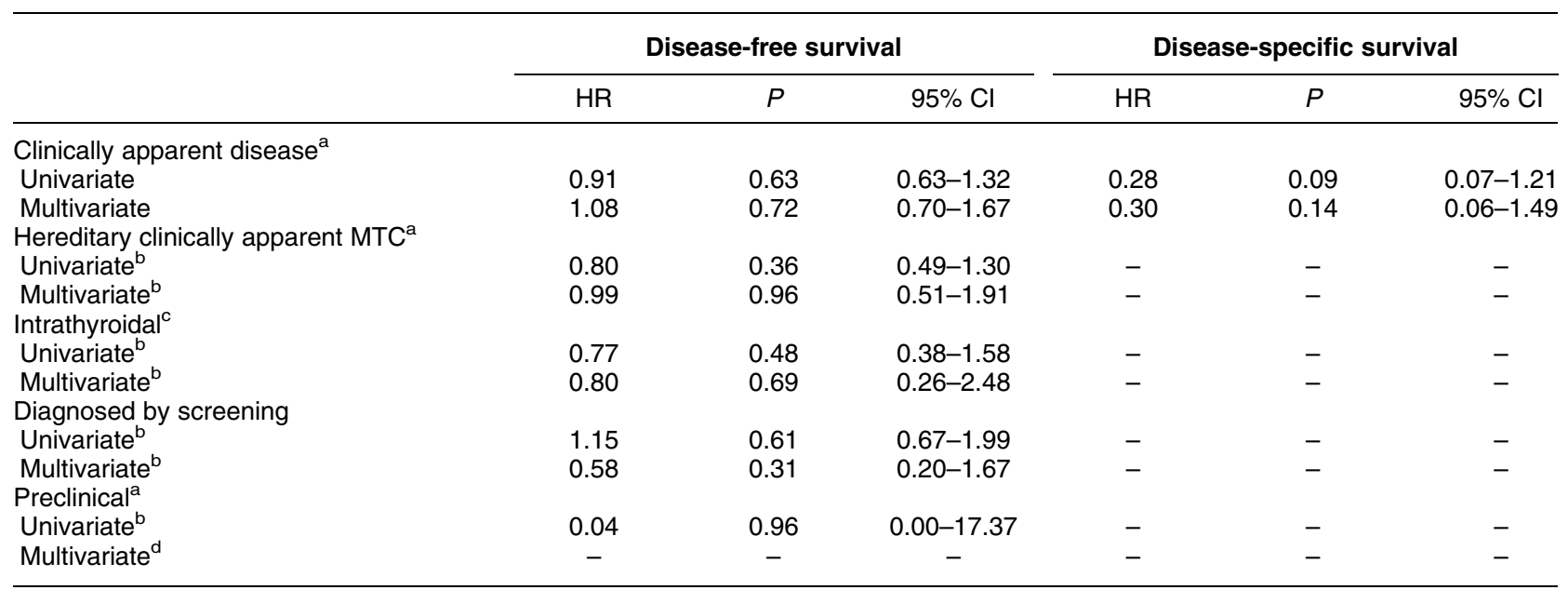

${ }^{a}$ Clinically apparent and preclinical disease was defined according to the ATA guideline of 2009.

${ }^{b}$ Owing to the limited amount of events disease-specific survival analysis was not possible.

'Localization: the 5th UICC edition was used to classify the MTC as intrathyroidal (T1-3N0) or extrathyroidal (T4 or N1).

${ }^{\mathrm{d}}$ Owing to the limited amount of events multivariate disease-free and disease-specific survival analysis was not possible. 
The following variables significantly influenced DFS of the included patients: hereditary stage $(\Delta \beta 57.8 \%$, i.e. the change in regression coefficient between the model with and without hereditary stage in the Cox regression analysis is $\% \Delta \beta 57.8 \%)$, center ( $\Delta \beta 128.9 \%$ ), extrathyroidal localization $(\Delta \beta 454.5 \%)$, and T stage ( $\Delta \beta 29.5 \%)$.

Inclusion of these confounders in multivariate analysis showed no significant difference in DFS between the two groups $(\mathrm{HR}=1.08 ; 95 \%$ CI $0.70-1.67$; $P=0.72$; see Fig. 2A).

Patients in the RAI group received a mean dosage of $2423 \mathrm{MBq}$ RAI (range 555-7400 MBq). In the Cox regression analysis, the dosage of RAI was not correlated with DFS (data not shown).

Hereditary clinically apparent MTC During the follow-up, remission was obtained in $20 \%$ of patients in the control group and $34.3 \%$ of patients in the RAI group $(P=0.058)$. Recurrence occurred in $9.3 \%$ and $17.1 \%$ in the control and RAI groups respectively $(P=0.06)$. Univariate analysis showed no significant difference in DFS for the two groups (HR $=0.80 \mathrm{HR}$ for RAI; 95\% CI 0.49-1.30; $P=0.36$; see Table 2). Also in multivariate analysis (corrected for $\mathrm{T}$ stage, center, extrathyroidal localization, adequacy of surgery, age, and gender), the difference in DFS between the two
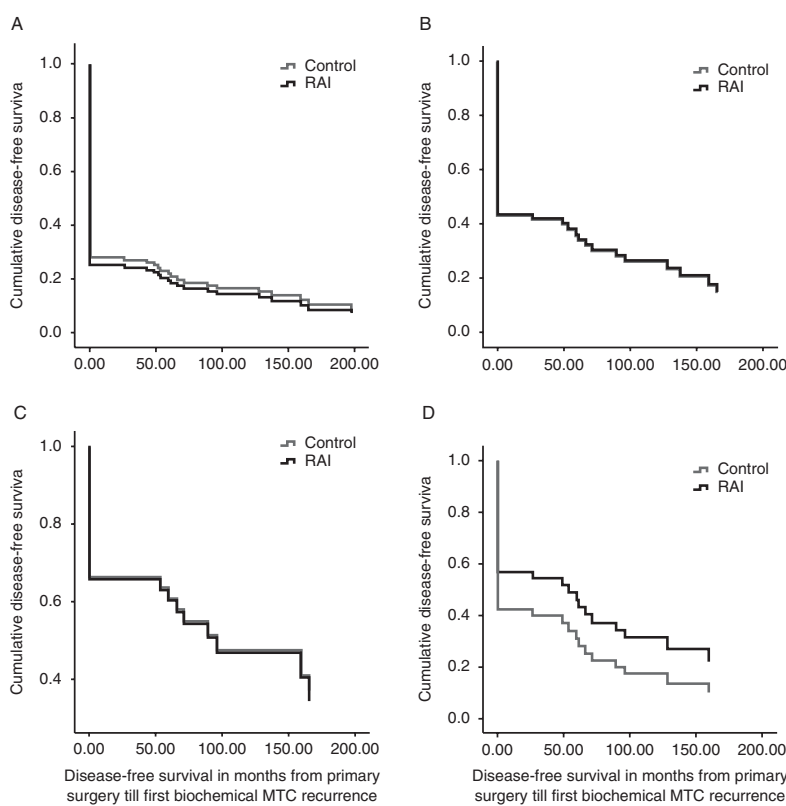

D

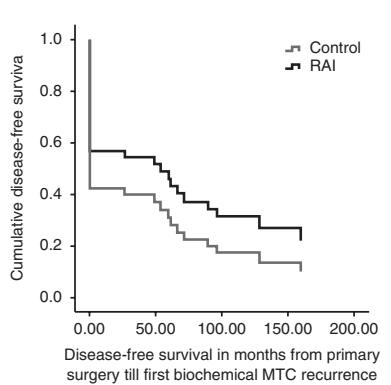

Figure 2 Cox regression analysis of the disease-free survival of patients with MTC. $Y$-axis, cumulative disease-free survival; $X$-axis, disease-free survival in months from primary surgery till first biochemical MTC recurrence. Control vs RAI treatment in patients with (A) clinically apparent MTC $(\mathrm{HR}=1.08 ; 95 \% \mathrm{Cl} 0.70-1.67$; $P=0.72)$; (B) hereditary clinically apparent MTC $(\mathrm{HR}=0.99 \mathrm{HR}$ for RAl; 95\% Cl 0.51-1.91; $P=0.96)$; (C) hereditary clinically apparent intrathyroidal MTC (HR=0.80; 95\% Cl 0.26-2.48; $P=0.69)$; and (D) hereditary clinically apparent MTC diagnosed by screening $(\mathrm{HR}=0.58 ; 95 \% \mathrm{Cl} 0.20-1.67 ; P=0.31)$. groups was not significant (HR $=0.99$ HR for RAI; 95\% CI $0.51-1.91 ; P=0.96$; see Fig. 2B).

\section{Subgroup analysis}

Localization In patients with hereditary clinically apparent MTC localized within the thyroid, univariate analysis $(\mathrm{HR}=0.77 \mathrm{HR}$ for RAI; 95\% CI $0.38-1.58$; $P=0.48$; see Table 2) and multivariate analysis, corrected for center, adequacy of surgery, $\mathrm{T}$ stage, gender, screening stage, and age, showed no significant difference in DFS (HR $=0.80 ; 95 \%$ CI $0.26-2.48$; $P=0.69$; see Fig. 2C).

Screening status Within the group of patients with hereditary clinically apparent MTC who were diagnosed with MTC by screening, DFS showed no significant differences between the two groups in univariate analysis (HR $=1.15 \mathrm{HR}$ for RAI; 95\% CI $0.67-1.99$; $P=0.61$; see Table 2) and multivariate analysis $(\mathrm{HR}=0.58 \mathrm{HR}$ for RAI; 95\% CI 0.20-1.67; $P=0.31$; see Fig. 2D), corrected for center, adequacy of surgery, extrathyroidal localization, age, and gender.

Preclinical MTC During the follow-up, remission was obtained in $60 \%$ of patients in the control group and $100 \%$ of patients in the RAI group $(P=0.14)$. Recurrence occurred in $6.4 \%$ in the control group and in none in the RAI group $(P=0.14)$. Univariate analysis showed no significant difference in DFS for the two groups $(\mathrm{HR}=0.04$ for $\mathrm{RAI}$; 95\% CI 0.00-17.4; $P=0.96$ ). Owing to the limited number of events, multivariate analysis of DFS was not possible.

\section{Disease-specific survival}

Clinically apparent MTC During the follow-up, 20 patients $(11.2 \%)$ died due to MTC in the control group and two patients (3.9\%) died in the RAI group $(P=0.13)$. Re-operations were undertaken in 55 patients $(30.7 \%)$ in the control group and in 18 patients $(35.3 \%)$ in the RAI group $(P=0.54)$. Univariate analysis showed no significant difference in DSS for the two groups $(\mathrm{HR}=0.28 ; 95 \%$ CI $0.07-1.21$; $P=0.09$; see Table 2). In mulivariate analysis, corrected for hereditary stage, extrathyroidal localization, $\mathrm{T}$ stage, and adequacy of surgery, DSS for the two groups was not significantly different $(\mathrm{HR}=0.30$; 95\% CI 0.06-1.49; $P=0.14$ ).

Hereditary clinically apparent MTC Five patients $(6.7 \%)$ died due to MTC in the control group and none of the patients died in the RAI group $(P=0.12)$ during the follow-up. Re-operations were undertaken in 23 patients $(30.7 \%)$ in the control group and in ten 
patients $(28.6 \%)$ in the RAI group $(P=0.82)$. Owing to the limited number of events (multivariate; subgroup), analysis of DSS was not possible.

Preclinical MTC As expected in this group, no patients died due to MTC. Two patients (4.2\%) underwent a re-operation in the control group and none in the RAI group $(P=0.53)$.

\section{Discussion}

In the present multicenter study, additional RAI treatment did not lead to a significant difference in DFS and DSS in patients with clinically apparent and preclinical MTC. Neither the stratification according to hereditary stage nor to tumor localization revealed significant effects of RAI treatment on the primary and secondary endpoints. Subgroup analysis of the dosage of RAI showed no effect on DFS either.

The overall DSS and the proportion of patients receiving complete remission in our study is consistent with other studies $(27,28,29,30)$. Compared with the literature, a high percentage of included patients had hereditary MTC $(47.8 \%$ of the patients with clinically apparent MTC). This is probably due to the fact that especially hereditary MTC patients are referred to tertiary centers. In addition, by excluding patients who had distant metastases or who had primary surgery without curative intent, particularly patients with sporadic MTC were excluded.

The absence of significant additional effect of RAI in the treatment of MTC shown in this study is consistent with the much smaller studies of Saad et al. (13) and Nieuwenhuijzen Kruseman et al. (14). Clinical studies on this topic are scarce and consist mainly of case reports. The added value of this study is the large number of patients treated with RAI, availability of a control group, long follow-up, and verification of the quality of surgery (see Supplementary Table 1, see section on supplementary data given at the end of this article).

A limitation of this study is its retrospective character. However, as the extent of surgery is an important aspect influencing outcome in MTC $(1,2,31)$, we evaluated this confounding factor in outcome by classifying the surgical interventions according to the ATA guideline of 2009. Although allocation of patients to RAI was not randomized, analysis did not show significant differences in the other prognostic parameters between the control and RAI group in general as well as within the hereditary group (see Table 1). There is no information in the literature on the level of absorbed beta radiation emanating from RAI in follicular cells that is likely to reach and destroy (clusters of) C-cells. The current dosage of RAI as given in differentiated thyroid carcinoma was not effective in MTC. Although subgroup analysis of the dosage of RAI showed no difference in DFS, we cannot exclude that higher than the current usual dosage of RAI might be effective in MTC. Nevertheless, inefficacy of radioiodine ablation might be due to the short range of beta emission and to the fact that MTC cells are rather resistant to irradiation.

In conclusion, in this retrospective controlled multicenter study, we found no significant additional value of radioiodine in the treatment of $\mathrm{MTC}$, which confirms the findings of previous studies. Based on the results of the present analysis, we endorse the current guidelines that RAI has no place in the treatment of MTC, either the hereditary or the sporadic form.

\section{Supplementary data}

This is linked to the online version of the paper at http://dx.doi.org/10. 1530/EJE-12-0943.

\section{Declaration of interest}

The authors declare that there is no conflict of interest that could be perceived as prejudicing the impartiality of the research reported.

\section{Funding}

This research did not receive any specific grant from any funding agency in the public, commercial or not-for-profit sector.

\section{Acknowledgements}

The authors thank Jean-Pierre Sels, Wouter Zandee, Stephan Hendriks, and Samuel Arends for their help in collecting the data.

\section{References}

1 Dralle H, Damm I, Scheumann GF, Kotzerke J, Kupsch E, Geerlings H \& Pichlmayr R. Compartment-oriented microdissection of regional lymph nodes in medullary thyroid carcinoma. Surgery Today 1994 24 112-121. (doi:10.1007/BF02473391)

2 Moley JF \& DeBenedetti MK. Patterns of nodal metastases in palpable medullary thyroid carcinoma: recommendations for extent of node dissection. Annals of Surgery 1999229 880-887. (doi:10.1097/00000658-199906000-00016)

3 Ukkat J, Gimm O, Brauckhoff M, Bilkenroth U \& Dralle H. Single center experience in primary surgery for medullary thyroid carcinoma. World Journal of Surgery $2004 \mathbf{2 8} 1271-1274$. (doi:10.1007/s00268-004-7608-9)

4 Kloos RT, Eng C, Evans DB, Francis GL, Gagel RF, Gharib H, Moley JF, Pacini F, Ringel MD, Schlumberger M et al. Medullary thyroid cancer: management guidelines of the American Thyroid Association. Thyroid 200919 565-612. (doi:10.1089/thy. 2008.0403)

5 Cupisti K, Wolf A, Raffel A, Schott M, Miersch D, Yang Q, Eisenberger CF, Röher HD \& Knoefel WT. Long-term clinical and biochemical follow-up in medullary thyroid carcinoma: a single institution's experience over 20 years. Annals of Surgery $2007 \mathbf{2 4 6}$ 815-821. (doi:10.1097/SLA.0b013e31813e66b9)

6 Dottorini ME, Assi A, Sironi M, Sangalli G, Spreafico G \& Colombo L. Multivariate analysis of patients with medullary thyroid carcinoma. Prognostic significance and impact on treatment of clinical and pathologic variables. Cancer 199677 1556-1565. (doi:10.1002/(SICI)1097-0142(19960415)77:8< 1556::AID-CNCR2O>3.0.CO;2-Y) 
7 Raue F, Kotzerke J, Reinwein D, Schröder S, Röher HD, Deckart H, Höfer R, Ritter M, Seif F, Buhr H et al. Prognostic factors in medullary thyroid carcinoma: evaluation of 741 patients from the German Medullary Thyroid Carcinoma Register. Clinical Investigator 199371 7-12. (doi:10.1007/BF00210956)

8 Bergholm U, Adami HO, Bergstrom R, Backdahl M \& Akerstrom G. Long-term survival in sporadic and familial medullary thyroid carcinoma with special reference to clinical characteristics as prognostic factors. The Swedish MTC Study Group. Acta Chirurgica Scandinavica 1990156 37-46.

9 Barbet J, Campion L, Kraeber-Bodere F \& Chatal JF. Prognostic impact of serum calcitonin and carcinoembryonic antigen doubling-times in patients with medullary thyroid carcinoma. Journal of Clinical Endocrinology and Metabolism 200590 6077-6084. (doi:10.1210/jc.2005-0044)

10 Gawlik T, d'Amico A, Szpak-Ulczok S, Skoczylas A, Gubała E, Chorazy A, Gorczewski K, Włoch J \& Jarzab B. The prognostic value of tumor markers doubling times in medullary thyroid carcinoma - preliminary report. Thyroid Research 2010310. (doi:10.1186/1756-6614-3-10)

11 Miyauchi A. Evaluation of surgical results and prediction of prognosis by analysis of serum tumor marker levels: proposal of a theory tested on patients with medullary thyroid carcinoma. Gan No Rinsho. Japan Journal of Cancer Clinics 198834 739-743.

12 Meijer JA, le Cessie S, van den Hout WB, Kievit J, Schoones JW, Romijn JA \& Smit JW. Calcitonin and carcinoembryonic antigen doubling times as prognostic factors in medullary thyroid carcinoma: a structured meta-analysis. Clinical Endocrinology 201072 534-542. (doi:10.1111/j.1365-2265.2009.03666.x)

13 Saad MF, Ordonez NG, Rashid RK, Guido JJ, Hill CS Jr, Hickey RC \& Samaan NA. Medullary carcinoma of the thyroid. A study of the clinical features and prognostic factors in 161 patient. Medicine 198463 319-342. (doi:10.1097/00005792-198411 000-00001)

14 Nieuwenhuijzen Kruseman AC, Bussemaker JK \& Frolich M. Radioiodine in the treatment of hereditary medullary carcinoma of the thyroid. Journal of Clinical Endocrinology and Metabolism 1984 59 491-494. (doi:10.1210/jcem-59-3-491)

15 Davis PW. A review of 17 cases of medullary carcinoma of the thyroid gland. Proceedings of the Royal Society of Medicine 1967 $60743-744$.

16 Faik EM, Gursoy A, Erdogan G \& Kamel N. Radioactive iodine treatment in medullary thyroid carcinoma. Nuclear Medicine Communications 200627 359-362. (doi:10.1097/01.mnm. $0000202860.30274 . e 4)$

17 Bayraktar M, Gedik O, Akalin S, Usman A, Adalar N \& Telatar F. The effect of radioactive iodine treatment on thyroid $\mathrm{C}$ cells. Clinical Endocrinology 199033 625-630. (doi:10.1111/j.13652265.1990.tb03901.x)

18 Lowery WD, Thomas CG Jr, Awbrey BJ, Rosenstein BD \& Talmage RV. The late effect of subtotal thyroidectomy and radioactive iodine therapy on calcitonin secretion and bone mineral density in women treated for Graves' disease. Surgery $19861001142-1149$.

19 Ott RA, Hofmann C, Oslapas R, Nayyar R \& Paloyan E. Radioiodine sensitivity of parafollicular C cells in aged Long-Evans rats. Surgery $19871021043-1048$.
20 Feinstein RE, Gimeno EJ, el Salhy M, Wilander E \& Walinder G. Evidence of C-cell destruction in the thyroid gland of mice exposed to high 131I doses. Acta Radiologica. Oncology 198625 199-202. (doi:10.3109/02841868609136405)

21 Michael BE, Forouhar FA \& Spencer RP. Medullary thyroid carcinoma with radioiodide transport. Effects of iodine-131 therapy and lithium administration. Clinical Nuclear Medicine 198510 274-279. (doi:10.1097/00003072-198504000-00010)

22 Shah KH, Oslapas R, Calandra DB, Prinz RA, Ernst K, Hofmann C, Smith M, Chejfec G, Lawrence AM \& Paloyan E. Effects of radiation on parafollicular C cells of the thyroid gland. Surgery 198394 989-994.

23 Deftos LJ \& Stein MF. Radioiodine as an adjunct to the surgical treatment of medullary thyroid carcinoma. Journal of Clinical Endocrinology and Metabolism 198050 967-968. (doi:10.1210/ jcem-50-5-967)

24 Hellman DE, Kartchner M, Van Antwerp JD, Salmon SE, Patton DD \& O'Mara R. Radioiodine in the treatment of medullary carcinoma of the thyroid. Journal of Clinical Endocrinology and Metabolism $1979 \mathbf{4 8}$ 451-455. (doi:10.1210/ jcem-48-3-451)

25 Engelbach M, Gorges R, Forst T, Pfützner A, Dawood R, Heerdt S, Kunt T, Bockisch A \& Beyer J. Improved diagnostic methods in the follow-up of medullary thyroid carcinoma by highly specific calcitonin measurements. Journal of Clinical Endocrinology and Metabolism 200085 1890-1894. (doi:10.1210/ jc.85.5.1890)

26 Twisk JWR. Multiple regressie-analyse: associatiemodellen en predictiemodellen. Inleiding in de toegepaste biostatistiek. In Inleiding in de toegepaste biostatistiek, edn 1, pp 229-273. Ed JWR Twisk. Amsterdam: Reed business, 2007.

27 Abraham DT, Low TH, Messina M, Jackson N, Gill A, Chou AS, Delbridge L, Learoyd D, Robinson BG, Sidhu S et al. Medullary thyroid carcinoma: long-term outcomes of surgical treatment. Annals of Surgical Oncology 201118 219-225. (doi:10.1245/ s10434-010-1339-y)

28 Ito Y, Miyauchi A, Yabuta T, Fukushima M, Inoue H, Tomoda C, Uruno T, Kihara M, Higashiyama T, Takamura Y et al. Alternative surgical strategies and favorable outcomes in patients with medullary thyroid carcinoma in Japan: experience of a single institution. World Journal of Surgery 200933 58-66. (doi:10.1007/s00268-008-9795-2)

29 Rendl G, Manzl M, Hitzl W, Sungler P \& Pirich C. Long-term prognosis of medullary thyroid carcinoma. Clinical Endocrinology 200869 497-505. (doi:10.1111/j.1365-2265.2008.03229.x)

30 Machens A, Hofmann C, Hauptmann S \& Dralle H. Locoregional recurrence and death from medullary thyroid carcinoma in a contemporaneous series: 5-year results. European Journal of Endocrinology 2007157 85-93. (doi:10.1530/EJE-07-0095)

31 Leggett MD, Chen SL, Schneider PD \& Martinez SR. Prognostic value of lymph node yield and metastatic lymph node ratio in medullary thyroid carcinoma. Annals of Surgical Oncology 200815 2493-2499. (doi:10.1245/s10434-008-0022-z)

Received 28 October 2012

Revised version received 12 February 2013

Accepted 5 March 2013 